\title{
Gestational Diabetes Mellitus and Pandemic Covid-19: Where do we Stand?
}

\begin{abstract}
Najmiatul $\mathrm{F}^{1,2 *}$
${ }^{1}$ Department of Pharmacology and Clinical Pharmacy, Universitas Andalas, Padang, Indonesia ${ }^{2}$ Unit of Pharmaco-Therapy, Epidemiology and -Economics, University of Groningen, Netherlands
\end{abstract}

*Corresponding author: Najmiatul Fitria, Unit of Pharmaco-Therapy, Epidemiology and -Economics, Faculty of Science and Engineering, University of Groningen, The Netherlands, Email: najmiatulfitria@phar.unand.ac.id

Abbreviations: GDM: Gestational Diabetes Mellitus; OADs: Oral Anti-diabetic Agents; OGTT: Oral Glucose Tolerance Test; HIC: High-Income Countries.

\section{Opinion}

Pregnant women with increasing blood glucose levels are identified as being more exposed to the severe effects of COVID-19 and advised to follow social distancing stringently. The COVID-19 pandemic has required rapid transformation and adaptation of healthcare services. Timely screening for hyperglycaemia in pregnancy using a simple glucose test still enhances early detection and control of gestational diabetes mellitus (GDM) [1].

Accumulated evidence has shown that hyperglycaemia during pregnancy not only increases perinatal morbidity and mortality in mothers and children but also increases the manifestation of disease later in life. The latest program to emphasized prevention and management of hyperglycaemia in pregnancy are:

- Planning and preparation before pregnancy; all women, especially women with diabetes, impaired glucose tolerance, impaired fasting glucose, and a history of GDM, need to plan for pregnancy and seek for pregnancy counselling early. Women with diabetes who are planning pregnancy and those using insulin should control their haemoglobin A1C (HbA1c) levels at $<6.5 \%$ and $<7 \%$, respectively, to prevent hyperglycaemia [2].

- Lifestyle modification; for women without contraindications to exercise, an accumulated time for moderate exercise of $\geq 30$ minutes on most, or at least 150 minutes/week. Sports involving risks or collisions should be avoided [3].
- Medical therapy in hyperglycaemia in pregnancy includes insulin and oral anti-diabetic agents (OADs) [4].

- Postpartum follow-up; the recommendation is to conduct the first postpartum follow-up at 6-12 weeks after childbirth. Additionally, breastfeeding can also reduce the risk of type 2 diabetes in women with hyperglycaemia in pregnancy [5].

The problem now is: How to access primary care during the lockdown situation? The impact of COVID-19 on antenatal glucose control will need to be carefully evaluated. Traditional midwifery topics such as support for the skin to skin contact for colostrum harvesting for management of neonatal hypoglycemia continue in online formats. Except in the dizziest women with COVID-19 infections (i.e., those requiring respiratory support or intensive care admission), the benefits of breastfeeding outweigh any potential transmission risks. The Baby-Friendly Initiative from UNICEF provides advice and support for women who are having difficulty accessing formula milk. The postnatal wards have benefitted from more bed space and fewer visitors, allowing a more comfortable postpartum experience than usual. Those attending pre-pregnancy clinics and (hopefully, most women with diabetes of reproductive years) were signposted to online resources or an interactive pregnancy planning tool [6].

Undertaking action has been done in this pandemic situation. It starts from diagnostic management of GDM during the pandemic, telemedicine to virtual care [7-9]. The critical point here is reducing contacts with health facilities. One of the strategies is changing the hospital visit time during the oral glucose tolerance test (OGTT). For example, pregnant women consume $50 \mathrm{~g}$ of glucose for GCT before 
Public Health Open Access

visiting hospitals, and blood samples are collected one $\mathrm{h}$ after consumption, it may shorten the amount of time required for them to be in a hospital.

Then we come to another problem. We do not have any equal facilities and excellent pre-ante natal services. It would not be a problem in high-income countries (HIC), but in this pandemic situation, everything becomes a problem. In France, they already build alternatives to GDM diagnostic, but some women do not wish to undergo the test [10]. In Japan, to prevent the COVID-19 infection, patients must minimize hospital visits, avoid spending long periods at the hospital, and limit face-to-face contact with healthcare practitioners [7]. However, GDM management during the COVID-19 situation would be a big challenges for non-HIC since there still many aspects that need to be established first. There is no published article found yet regarding GDM management in LMIC during the COVID-19 pandemic.

\section{References}

1. Fitria N, van Asselt ADI, Postma MJ (2019) Costeffectiveness of controlling gestational diabetes mellitus: a systematic review. Eur J Health Econ 20(3): 407-417.

2. (2014) Diagnosis and Therapy Guideline of Pregnancy with Diabetes Mellitus. Zhonghua Fu Chan Ke Za Zhi 49(8): 561-569.

3. (2020) Physical Activity and Exercise During Pregnancy and the Postpartum Period. Obstet Gynecol 135(4): 991-
993.

4. (2013) WHO Diagnostic Criteria and Classification of Hyperglycemia First Detected in Pregnancy. World Health Organization, pp: 66.

5. Gunderson EP, Lewis CE, Lin Y, Sorel M, Gross M, et al. (2018) Lactation Duration and Progression to Diabetes in Women Across the Childbearing Years: The 30-Year CARDIA Study. JAMA Intern Med 178(3): 328-337.

6. Murphy HR (2020) Managing Diabetes in Pregnancy Before, During, and After COVID-19. Diabetes Technol Ther 22(6): 454-461.

7. Kasuga Y, Saisho Y, Ikenoue S, Ochiai D, Tanaka M (2020) A new diagnostic strategy for gestational diabetes during the COVID-19 pandemic for the Japanese population. Diabetes Metab Res.

8. Peahl AF, Smith RD, Moniz MH (2020) Prenatal Care Redesign: Creating Flexible Maternity Care Models Through Virtual Care. Am J Obstet Gynecol pp: 1-21.

9. Aziz A, Zork N, Aubey JJ, Baptiste CD, D’Alton ME, et al. (2020) Telehealth for High-Risk Pregnancies in the Setting of the COVID-19 Pandemic. Am J Perinatol.

10. Vambergue A, Jacqueminet S, Lamotte MF, Lamiche LF, Brunet C, et al. (2020) Three alternative ways to screen for hyperglycaemia in pregnancy during the COVID-19 pandemic. Diabetes Metab. 